\title{
Creating social, physical, and authoring games
}

\author{
Paul Lapides \\ University of Calgary \\ 2500 University Drive NW \\ Calgary, AB, Canada T2N 1N4 \\ paul.lapides@gmail.com
}

\author{
Ehud Sharlin \\ University of Calgary \\ 2500 University Drive NW \\ Calgary, AB, Canada T2N 1N4 \\ ehud@cpsc.ucalgary.ca
}

\author{
Mario Costa Sousa \\ University of Calgary \\ 2500 University Drive NW \\ Calgary, AB, Canada T2N 1N4 \\ smcosta@ucalgary.ca
}

\begin{abstract}
Computer games have become increasingly cooperative social activities that are often being played with several collocated gamers, frequently in front of spectators who react and participate in the gameplay experience. Physical controllers are enriching the gaming interface by allowing players to use their bodies to control the game. We highlight another gameplay theme: in-game authorship of content, the ability to create new expressive content as part of the gameplay.

We propose a new design framework for cooperative games that encompasses these three themes: social engagement between players, expressive physical interaction within the game environment, and in-game creation and authoring of sharable content. These elements may be combined in various ways, and we propose prototypes for three games based on these themes: a comic strip game, a movie karaoke game, and a virtual competition game.
\end{abstract}

\section{Keywords}

Cooperative games, content authorship, physical interfaces, social interfaces, computer entertainment, video games, humancomputer interaction.

\section{INTRODUCTION}

The video game industry has expanded tremendously in the last decade; four times as many game units were sold in 2008 than in 1996. Predictably, the gender and age demographics have also changed. Today, video games are played by both genders $(40 \%$ are female) and by all ages (25\% are 18 and younger, $26 \%$ are 50 and older) [3]. Video games are often (and incorrectly) thought to be isolating activities, but in fact $62 \%$ of gamers in the USA play with other gamers in person. This is reflected in the type of platform the majority of games are sold for. Over $60 \%$ of US households own a computer and only $42 \%$ own a console, yet over $90 \%$ of game units sold in 2008 were developed for a game console $[3,11]$. Compared to the computer, consoles are better suited for games with multiple gamers because they are typically played on a large screen in a more open space. This allows players to enjoy the game while others may spectate while waiting their turn to play, or simply to watch and socialize.

Recently, game systems are offering players new controller options that allow more physical interaction when playing the game. The top four best selling games in 2008 were developed for

Permission to make digital or hard copies of all or part of this work for personal or classroom use is granted without fee provided that copies are not made or distributed for profit or commercial advantage and that copies bear this notice and the full citation on the first page. To copy otherwise, or republish, to post on servers or to redistribute to lists, requires prior specific permission and/or a fee.

CGAT Conference 2010, April 6-7, 2010, Singapore.

Copyright 2010 CGAT the Wii [3], a console system that uses wireless controllers that gamers can physically move with their hands, rather than pressing buttons and joysticks as the primary interaction method. Gamers can purchase specialized controllers in the shapes of steering wheels, guitars, and even a pad, the WiiFit, that senses the player's overall body mass movements. In addition to playing with other people, gamers are purchasing systems that allow them more physical interaction with the game [14, 15].

In this paper we will discuss the game design elements of social and physical gaming and how these are combined in current video games, as well as in the games we designed. A third element, user generated or authored content, which has seen limited use in games, is identified and explored.

\section{RELATED WORK}

Pervasive and physical games have been studied in the HumanComputer Interaction community [5]. Bjork et al. describe Pirates!, a multiplayer sea-faring game played on mobile devices in a large physical space [1]. Players can freely move around to different physical locations tagged with proximity beacons. When a player's mobile device activates a beacon, their in-game location moves to reflect the change in physical location. Each player controls a ship that gathers at islands, ports, and even at open sea for battle. The physical control of the game means that the players themselves meet at certain physical locations. The researchers found that spontaneous social interaction occurred between players as they gathered and moved in the physical space. This socialization was not mandated by the game but emerged naturally.

Sall and Grinter study the uses and practices of physical games in the home [9]. Many participants in their study purchased physical games as a form of exercise. Dance Dance Revolution, originally an arcade game, allows players to use their body to play by stepping on colored pads on the ground in synchronization to scrolling colors on the screen and a music track. The intense physical work needed to play the game is an effective workout, and while the initial motivation to play games like DDR is for exercise benefits, many users began playing the game for fun. Participants in the study refer to physical games as "performances". People not actively playing the game began to participate by becoming spectators that cheered players on and were engaged by the performance. In fact, the study participants reported that the lack of spectators made playing the game seem "weird" and not as fun.

The spectator experience is a phenomenon Reeves et al. examines and classifies [8]. Rather than categorizing interaction as public or private, Reeves et al. deconstructs interaction into manipulations and effects. In this taxonomy, manipulations are described to be input as well as non-input actions made by the user (or performer). These non-input actions can be gestures, speech, movements, glances, and may be done for technical reasons, such 
as picking up a new interface or adjusting an interface for more comfortable use, or for artistic purposes, such as timing users' movements to music for a more aesthetically pleasing performance. Effects are the results of these manipulations and may include the presentation or modification of images, graphics, and sounds, as well as changes to the interface itself such as menus, icons, and cursors. Manipulations and effects can each be classified in terms of their visibility: hidden, partially hidden, revealed, and amplified. This classification creates a relationship between the visibility of a manipulation and its effect, and the visibility of each may be changed based on the performances' needs. For example, a PowerPoint presentation will have partially hidden manipulations (mouse clicks) but amplified effects (clearly changing visuals). Four types of general approaches that may be used to create an interface are identified: secretive (hidden manipulations and effects), expressive (amplified manipulations and effects), magical (hidden manipulations and amplified effects), and suspenseful (amplified manipulations and hidden effects).

Voida and Greenberg treat console games as computational meeting places and study the social dynamics surrounding them [12]. As in Sall and Grinter's research, Voida and Greenberg report that gamers feel "lonely" when playing by themselves; mature and elder gamers seldom play alone. In addition to the social company of playing with others, the resulting interactions between gamers such as teamwork, common goals, and shared success were particularly motivating. Competitive spirits were also observed, and players sometimes enjoyed watching their friends fumble with the game and made light-hearted jokes with each other. Finally, a comparison between social in-person gaming and online multiplayer gaming is made. In the latter case, the gamer is playing while still isolated from anybody else; they play the game surrounded by other players, but not necessarily with the other players.

\section{DESIGN FRAMEWORK}

Our game design framework is based on identifying and employing three gameplay elements which we believe can create more engaging and fun collaborative games: sociability,

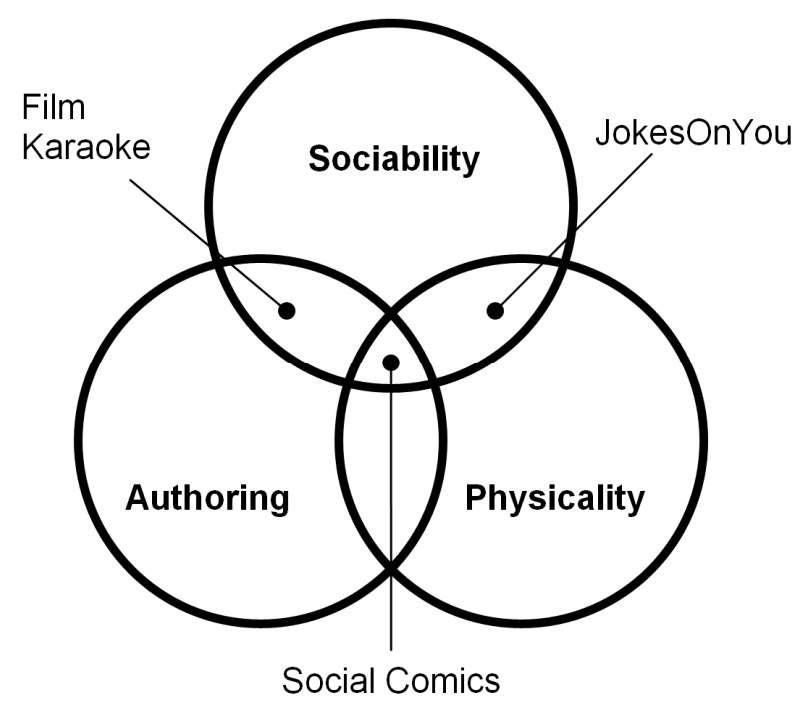

Figure 1: Sociability, physicality, and authorship. physicality, and authoring of content (Figure 1). We will examine how these three elements can be combined and used in defining a design framework for games. By choosing to incorporate various amounts of each element in a game, we can design games for different settings and possibly different demographics of gamers.

\subsection{Authorship of Content}

The games that we design will have a strong element of authorship. This is when one of the primary gameplay features is for the players to generate new content, whether it is photos, videos, audio, or some other form of media. Several games allow players to personalize various gameplay features, such as customizing a car in a racing game, or creating an avatar for roleplaying games. While this is a form of authorship, we argue that these features are most often secondary to the main gameplay experience. The player can still race in generic cars, or go on missions using generic characters; the racing and missions are the primary goals of the game in these instances, and the authoring is not a dynamic gameplay component.

Spore is one of a few games with authorship at the center of the gameplay [16]. In Spore players control an organism and help it evolve through different stages of life (micro-organism, animal, tribal, city, and interplanetary stages). Players must decide how their creature will look, behave, and interact with its environment. These are not simply cosmetic decisions but influence how the creature moves, eats, and even mates; in later stages it influences how the creatures behave politically and socially with one another. The authorship component is central to the gameplay of Spore. The player's creature is uploaded to a server and asynchronously distributed to other players' clients, populating the worlds that their own creatures live in.

We see great potential in exploring the element of gameplay authoring, and see its inclusion in the games we design as crucial. Authoring gameplay is not trivial to design. It must be an engaging experience but may lack explicit goals. In the case of Spore, the players may want to create an organism that is bizarre or unique while fulfilling the organism's survival goals of defense or predation; these are both implicit goals, though the latter is more important to the success of player.

Popular customization interfaces may include buttons, sliders, color wheels, etc. The player should be motivated to use this interface by receiving a continuous preview of their content as they make changes. In Spore, the walking behavior of the creature changes in real-time as the user adjusts the size of the legs, for example. A preview system such as this encourages the player to creatively explore the interface. One important feature of the interface is that the adjustments are imprecise. The player should not be burdened with measurements or numbers, unless absolutely necessary. The goal of our games is not for the player to create precise content but instead to creatively explore possibilities as if in a sandbox or an improvisational jam session.

\subsection{Physicality}

When we discuss physicality, we are talking about games where the players use their bodies to control the game. To some extent players use their bodies whenever they use game controllers, but this interaction metaphor often relies on complex mapping of buttons and joysticks to specific gameplay actions. We believe that including expressive physical interaction will simplify the learning curve of the game; players can quickly have fun with the game without spending many hours learning the controls. We want to let the player use movements and gestures that they 


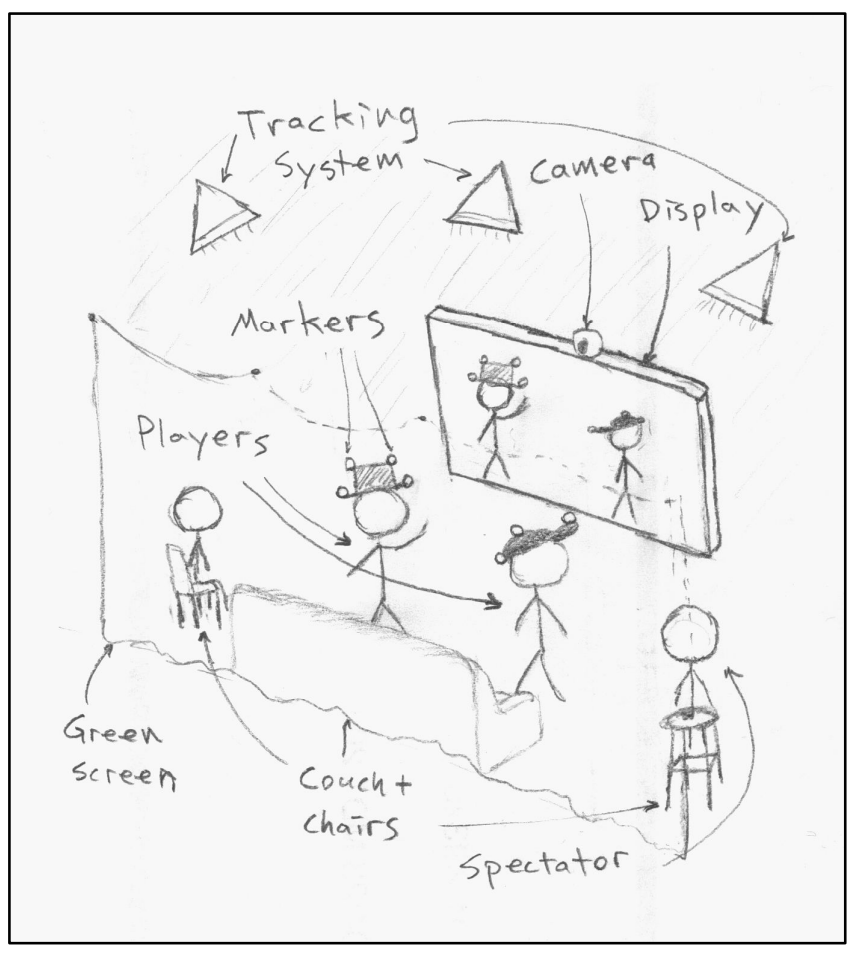

Figure 2: System overview sketch.

already know from other experiences to control the game. For instance, instead of pressing the "jump" button, the player can physically jump into the air. To push this interaction metaphor further, we envision ordinary physical objects becoming game entities. These artifacts have physical affordances that will make them intuitive to use in the game. A physical ball can be represented as a game entity, but it can also be thrown to another player. The players are not using a controller to throw a virtual ball, but the real ball has now become the controller itself.

Expressive physical actions such as jumping are required and normal when playing the game, but they may appear odd in nongaming situations. It is important for the players to feel comfortable in the social setting so that they can make these physical gestures and movements that may otherwise be perceived as odd. If the players are strangers, or if non-participating observers are present, the players may hesitate to play using physicality.

\subsection{Sociability}

We focus our game design on collocated games primarily because online games typically do not support rich communication between players. Screen chatting tools are slow and do not convey non-verbal communication. While voice chat allows tonal and vocal cues to be communicated, it fails at transmitting important physical cues between users. Finally, the fact that players are online imposes an anonymity and separation between users. This can lead to aggressive behavior, trolling, and spamming, often with no consequences.

We recognize that playing games together with collocated people allows natural communication between players, and usually imposes healthy social etiquette upon the gamers. However, being collocated is not enough; the participants must be actively engaged in playing the game. If one person is playing and another

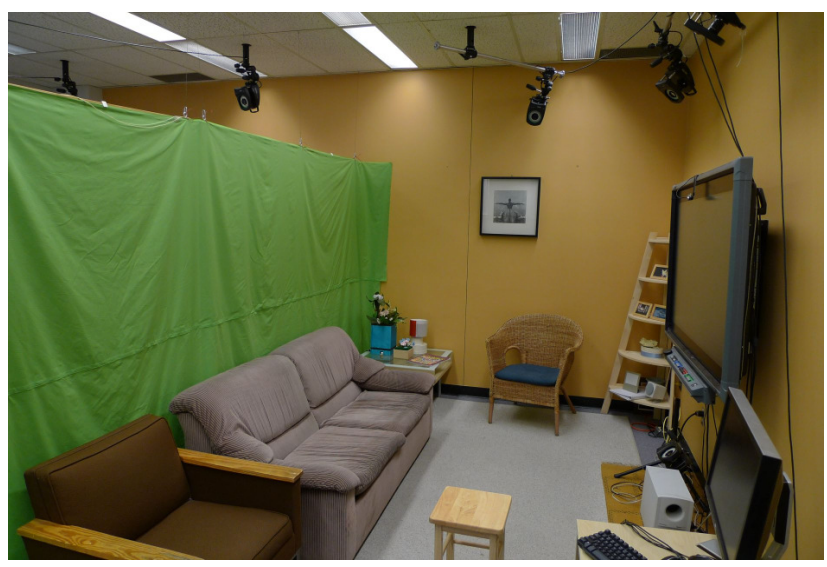

Figure 3: Green screen, display, and Vicon cameras.

person is reading a book or distracted with some other task, then this is not social gaming. Both people should be engaged with the game. In our design framework, sociability is when games are played with collocated and active participants.

When we consider the game participants, we look beyond the explicit players themselves. While all the game participants should be engaged in the gameplay, they do not need to be directly playing. Participants can be part of an audience that is equally engaged in the game as the currently players. If designed well, spectating is a fun experience in its own right; the audience can provide support, challenge, and conversation to the players.

\subsection{Summary}

We have discussed three design elements and how we wish to integrate them into our games. The individual elements may have been explored and implemented in current games. However, we believe that the amalgamation of all three elements together will create a novel, fun, and engaging gameplay experience.

\section{SYSTEM OVERVIEW}

The game prototypes that we describe will be played in a home theatre setting similar to the setting in which console games are played. The system described in this section is common to all three game prototypes.

\subsection{Environment}

Players will be in an open space in front of a wall-mounted display (Figure 2 and 3). The games will be played in a standing position, as players will typically use their entire bodies. However, there will be a couch and a series of chairs several meters from the display so the players can relax, and so spectators may watch the game being played from the side.

Above the display is a small camera pointed at the players. The image from the camera will be shown horizontally mirrored on the display, so the players will be able to see themselves as they move in the space, similar to the PlayStation Eye or EyeToy [13]. While this camera is primarily used to give video feedback to the players, in some instances it is used for tracking game objects, described later.

Behind the couch hangs a large sheet of green fabric, commonly called a "green screen". This enables simple isolation of the foreground players from the background of the room by using a straightforward chroma-key filter to remove the shade of green in the fabric [2]. A static or dynamic image is used to replace the 


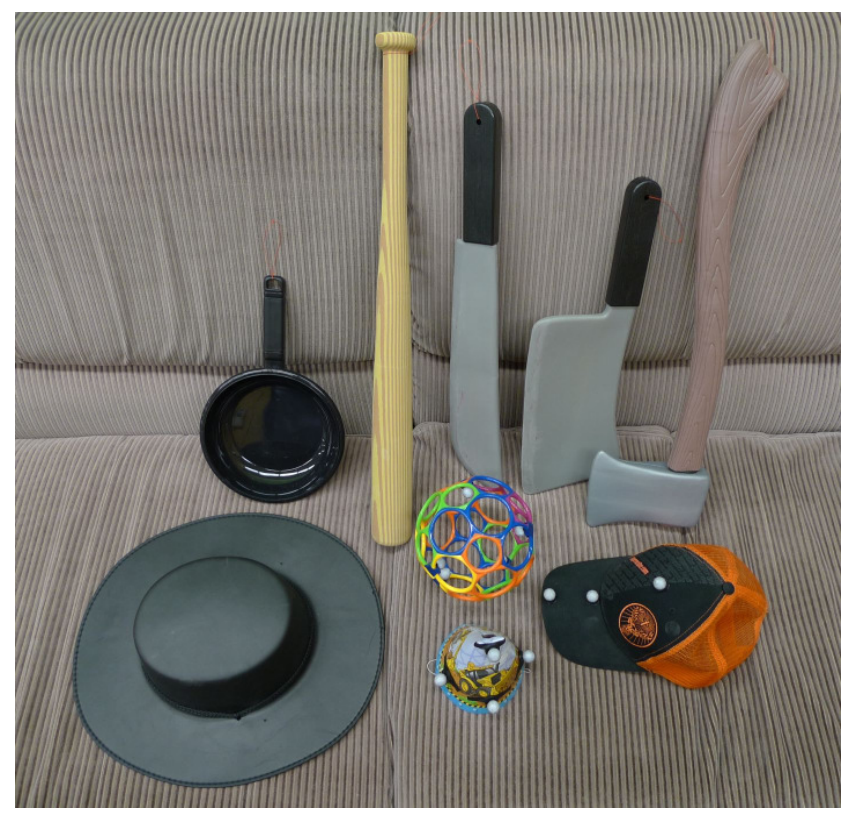

Figure 4: Hats and other props used in the games.

green color that is removed, resulting in an augmented reality image of the players in front of a different background. We chose this simple approach although more complicated algorithms may be used in the future without a green screen.

Our environment includes a series of props that may be used when playing the games (Figure 4). Some of these props are used mainly for tracking purposes while the others are used mainly for creative, expressive and aesthetic qualities. For example, there are a number of hats that the players can wear that are used to track the position of the players' heads. There are also a number of plastic swords, replica sports equipment such as a foam baseball bat and plastic hockey sticks, and miscellaneous props like a ball, hoola-hoops and frying pans. These props are used mainly for creative physical expression and may be tracked depending on their uses in the game being played.

\subsection{Tracking}

Our system does not use controllers, keyboards, or mice but instead relies on physical interaction as the basis of player input. Therefore, we must use a very robust and flexible system for tracking the three dimensional position of the players' bodies.

For the majority of the game tracking, we use a Vicon motion capture system. This is an optical tracking system that uses multiple cameras positioned on the outside of the tracking space that detect spherical markers. Each camera has a powerful infrared emitter that floods the space with IR light. The markers are specially coated to reflect this wavelength back to the cameras, which have an IR filter of the same wavelength in front of the sensor. The cameras produce a raw grayscale image that only includes the reflections from the markers, which appear as white circles on a black background. At every sample interval, each camera produces an image that is segmented, processed, and fed into an algorithm that determines the 3D position of each marker in the tracking space. The accuracy of this system is capable of detecting motion smaller than $1 \mathrm{~mm}$ at very high sampling rates, up to 200 samples per second. For our needs, typically 6 or more cameras are required to reduce errors from occlusions, where one or more cameras cannot see a marker because it is hidden behind an object.

This system is very popular in the film industry for recording complex human motion that is later used to create special effects. Videos game designers also use the system to create life-like animations for virtual characters. Both of these methods record a motion in order to process it, offline, in the future. We do not record motion but use the Vicon system in real-time for input, a technique that has become commonly used in the HCI community [4].

The spherical markers can be attached using adhesive tape or glue to any object, including the player's body. Three or more markers are required to accurately track an object. Multiple objects may be tracked in the space, as long as they are tagged with a unique orientation of markers. For example, a wooden rod may have two markers on each end and a marker in the middle, forming a line. A baseball hat may have three markers arranged in a triangle. The shapes created by the markers are unique, and the system is able to differentiate between the rod and the hat, even though both use three markers each, due to the different topologies of markers. The markers are light and unobtrusive, allowing us to track the player's head (hat), hands (gloves), even feet and torso, and any prop that we decide to include as an interaction mediator in the gameplay.

Currently, it is not realistic for the Vicon system to be used in a real console game setting because of its prohibitively high cost. We use this system to demonstrate new physical interaction methods that we believe can be implemented in the near future with much less expensive tracking hardware $[14,15]$.

A second method of tracking that our system utilizes is based on the camera above the display. This is a computer vision based tracking system that detects black and white barcode tags that are $8 \times 8$ grids with a different combination of black and white cells capable of encoding a unique number. The position and orientation of the tag relative to the camera can be extracted and used to overlay graphics above the tag. The quality of the camera and distance of the tag from the camera influence how accurate the tracking is. A small tag far away from the camera may not even get recognized as a tag. This approach is commonly used in augmented reality applications, and there are several robust libraries used for tracking, such as the ARToolKit+ library that we use

The objects that this system will track are large postcards that will be used to change the background. Each postcard will have a picture of a different setting or location corresponding to a digital background on one side and a large 2D tag on the other. When the players hold the postcard up to the camera, they will see the picture on the postcard while the camera will see the tag on the reverse. This mechanism allows the players to change the setting in several of our game environments to the one presented in the postcard.

\section{GAME INSTANCES}

In this section we present three novel games that use varying degrees of the three design elements presented in our framework (Section 3): sociability, physicality, and authorship of content. We will discuss how these game instances incorporate the design elements into their gameplay, describe our design considerations, the games low-fidelity prototypes, and the implementation challenges. 


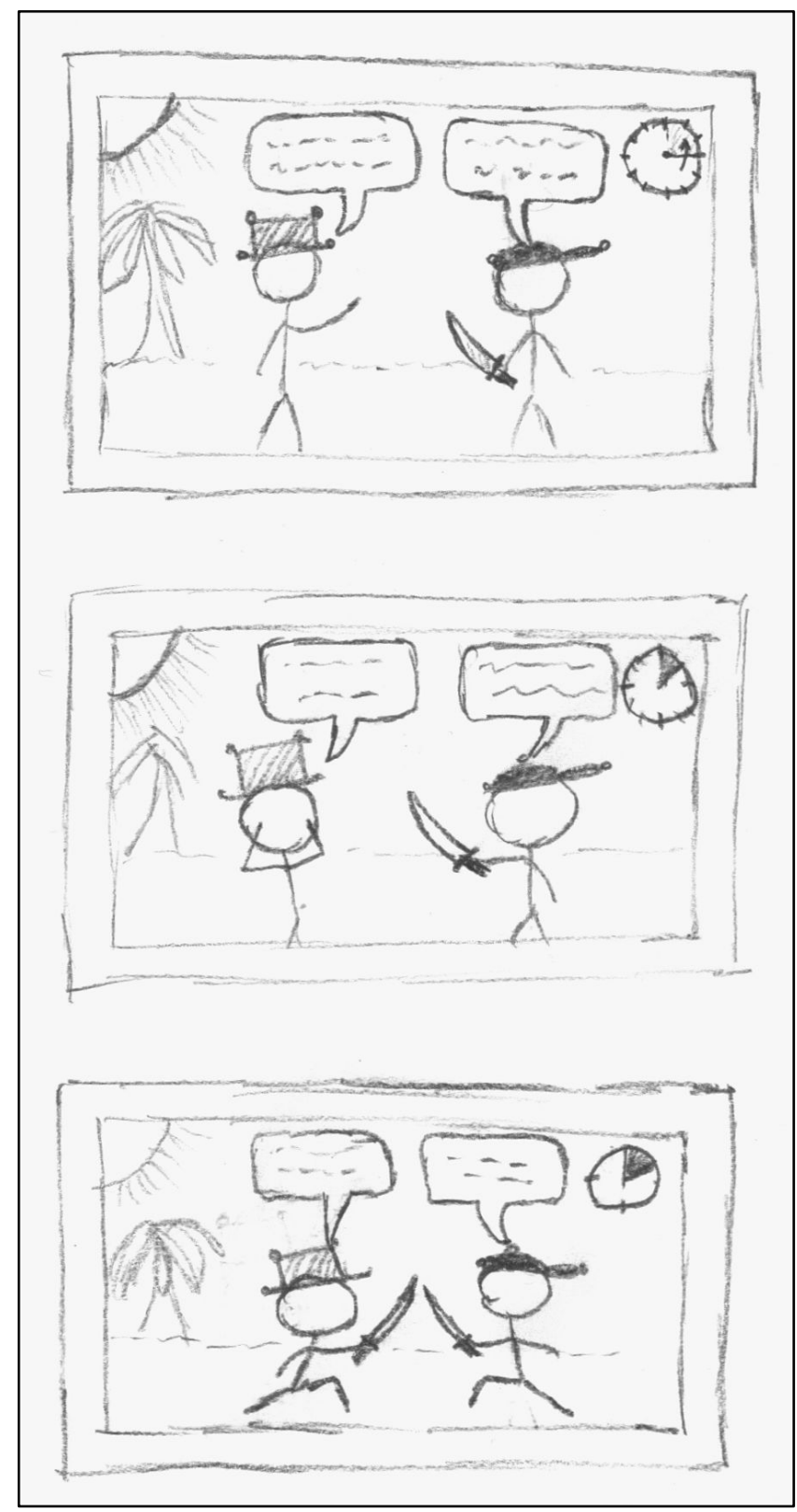

Figure 5: Sketch of the stages of the gameplay in Social Comics.

\subsection{Social Comics}

The first game we designed based on our framework is called Social Comics and incorporates all three design elements into its gameplay (Figure 5). This game is played with a group of people, though optimally only two players interact with the game directly at one time, while the others spectate and wait for their turn.

The goal is for the two players to create a humorous comic strip, only three panels long, in a limited amount of time. Before the game begins, the players' mirror image is shown on the display in front of the green background and the players can use the postcards to change the setting of their comic. Possible settings may include a street, office, park, open field, or more bizarre locations like the surface of the moon, under the ocean, or a volcano. Players should also wear a hat (from a variety of
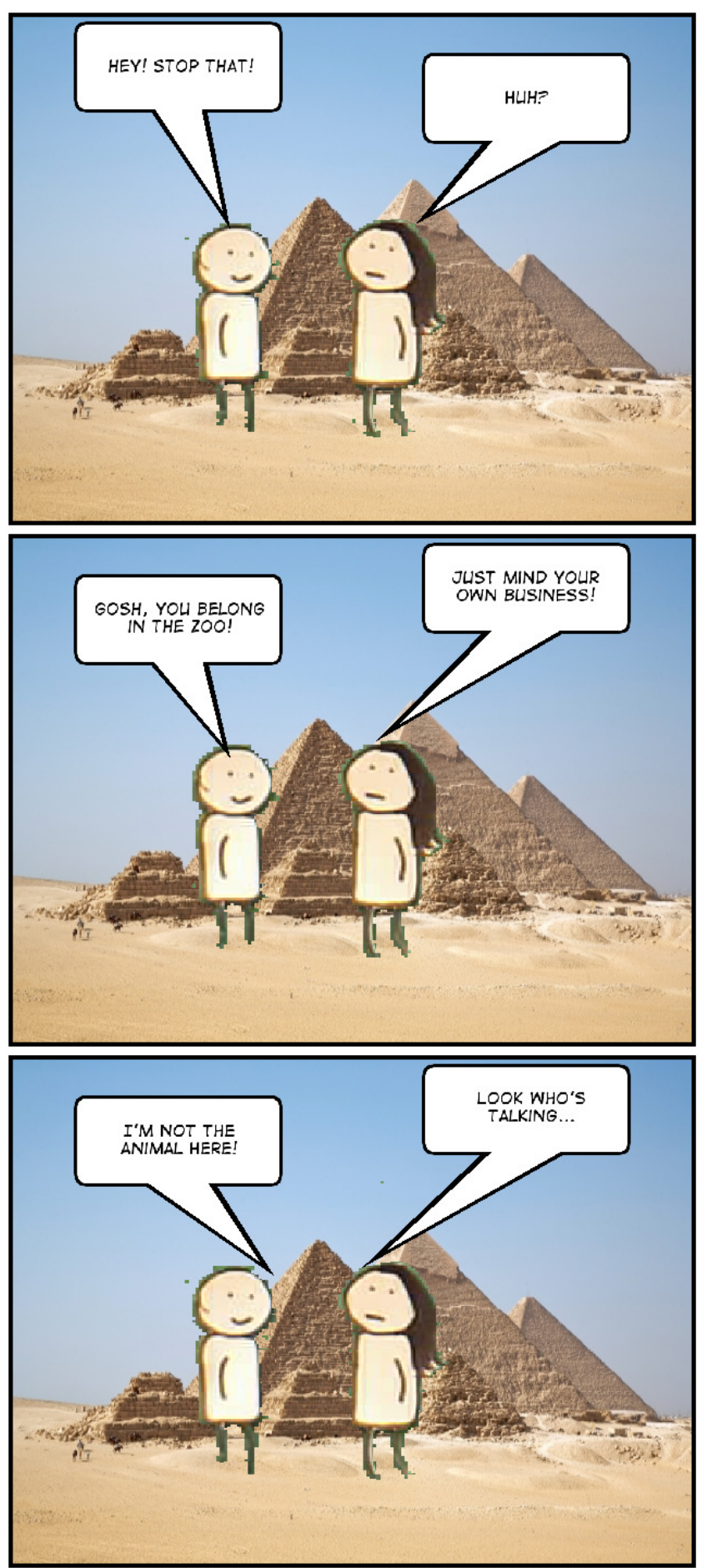

Figure 6: Medium-fidelity interactive prototype of Social Comics.

available hats) that is necessary to track the head of each player. The props can be used as well, and while they are not necessary for the game to function, both the hats and props may help the players appear and act like a particular character in the comic.

When the game begins, the players will have 10 or 15 seconds to decide what will happen in the first panel of the comic. A virtual clock will count down the time and the camera above the display 
will take a photo when the clock reaches zero. This photo will include everything that is shown on the screen (except the clock): the players, hats, props, and the setting they have chosen. The clock will reset and the players will decide what happens in the second panel of the comic. The clock will reset a third and final time for the last panel of the comic strip. The photos taken for each panel are assembled together and create a comic strip (Figure $6)$.

In this case, the comic strips will only have three panels, but this can change. We chose three panels because we wanted each comic to be finished quickly. Creating a longer comic with a story line may not be feasible given the limited time allocated to the players. In fact, the players may have difficulty creating even a simple story with accompanying visuals with such narrow time constraints.

We recognized that providing no direction or goal to the comic might overwhelm some players. Asking someone to create a funny story, let alone a visual comic strip and to illustrate it in 45 seconds may be infeasible for many people. We wished to provide some guidance in the creation process, but ultimately allow the players to decide what comics to create. Since our gameplay revolves around creating comics, we are exploring the use of speech bubbles as game elements.

Text and speech bubbles are widespread story telling tools in comic strips [6]. We use speech bubbles to give each player a simple line of dialogue in each panel; six lines of text per strip. The text appears on the display inside a white bubble and an arrow points from the text to the player's head, using the Vicon system and marked hat to track the player's position on the screen. The text must be carefully selected so that it does not explicitly tell the players what to do, but merely suggests a possible scenario for them to act out. It is designed to be vague and may be interpreted in many different ways by the players. The text for each comic is arranged to support a simple story unfolding throughout the panels; each piece of text has its specific place in the comic strip.

Once the players complete a comic strip, it is saved to the hard drive and is displayed on the screen so the audience may view the entire comic in its entirety. The saved strips can be reviewed later on the screen, printed, or shared online on social networking sites.

Social Comics incorporates the three design elements discussed earlier. Players interact socially to discuss their comic strategy with each other and also with the spectators, engaging both groups of game participants. The game is played by physically moving around, making poses, and using props to create each comic panel. Finally, while the game is being played, the players create new comic strips that can be viewed and shared.

\subsection{Film Karaoke}

The second game we are designing is a karaoke style game applied to film instead of music. The game is played in the same environment as Social Comics with a group of people (active players and spectators).

The goal of Film Karaoke is to recreate famous scenes from movies or TV shows. The players can select the scene they want to recreate from a list of offered choices. Note that the scenes the game will offer need to be very carefully chosen by the designers. A scene should be easily recognizable and based on a movie, TV show, or theatrical play that is part of popular culture. The scene should also include two or three characters engaged in dialogue with one another. Finally, the scene should be self-contained (e.g. not requiring extensive knowledge of the overall story of the film) and relatively short, around 2-4 minutes long.

The players will recreate the scene by each acting the part of one of the characters. The gameplay is based on replacing, in realtime, the original characters' faces in the scene with the faces of the players. The scene is shown on the display and each player will say their designated character's dialogue. The players will also act out the facial expressions of their characters, as their faces are superimposed in real-time to the scene. A microphone will record the lines being read by the players. When the scene is finished, it will include the character's original motions and actions, but with the faces, voices, and dialogue replaced by the corresponding player's face and voice.

The game isolates each player's face from the camera's image, so the players must be facing forward. Each player that is acting will wear a hat, but unlike Social Comics, these hats are only used for tracking purposes rather than having an added theatrical expressive role. Once the position of each player's head is known, the face can be isolated into its image by copying a region of the camera's image that corresponds to the position of the head in 3D. While we could have used facial detection algorithms for this process, we believe that the Vicon system will provide simpler and more robust tracking for our prototype game. Subtitles are shown under the scene to help the players with the dialogue but there is no restriction to what the players say in the scene. The players may decide to ignore the subtitles entirely and replace it with their own dialogue.

When a scene is finished, a new version of the original video is created, one that is based on the player's recorded audio dialogue and the player's facial expression as they acted and read the dialogue. The new scene is saved as a video file on the hard drive and may be watched on the display or uploaded to a video sharing website or other social networking tools.

We believe that careful scene selection is crucial to the success of the Film Karaoke game. Popular film and TV scenes are often alluded to in conversation by people imitating the characters or using direct quotes. If a scene is unfamiliar, players may not feel motivated to act in it because they may not relate to the characters. Scenes with two or three active characters may be simpler to act in and less confusing for the players than complex scenes with a lot of dialogue. The players will be more likely to watch or share their recreated scenes if the scene is short and does not rely on previous knowledge of the story.

Film Karaoke is an authoring game at its core. The goal of the game and what it creates is clearly defined. However, the game also has a significant social component. Players have to engage each other with dialogue, gaffs in dialogue will lead to humorous situations, and the recreated scenes are watched and shared among friends. The physical expression element of this game is arguably weaker, as players are not using any expressive artifacts during gameplay, other than their faces.

\subsection{JokesOnYou}

JokesOnYou, a two player competition/fighting game also uses our unified environment (Section 4). In this game, both players use augmented reality to compete and embarrass their opponent.

Two people will play standing up in front of the display, which mirrors their motions as in Sociable Comics. The players will wear marked hats and gloves, enabling the system to track their 


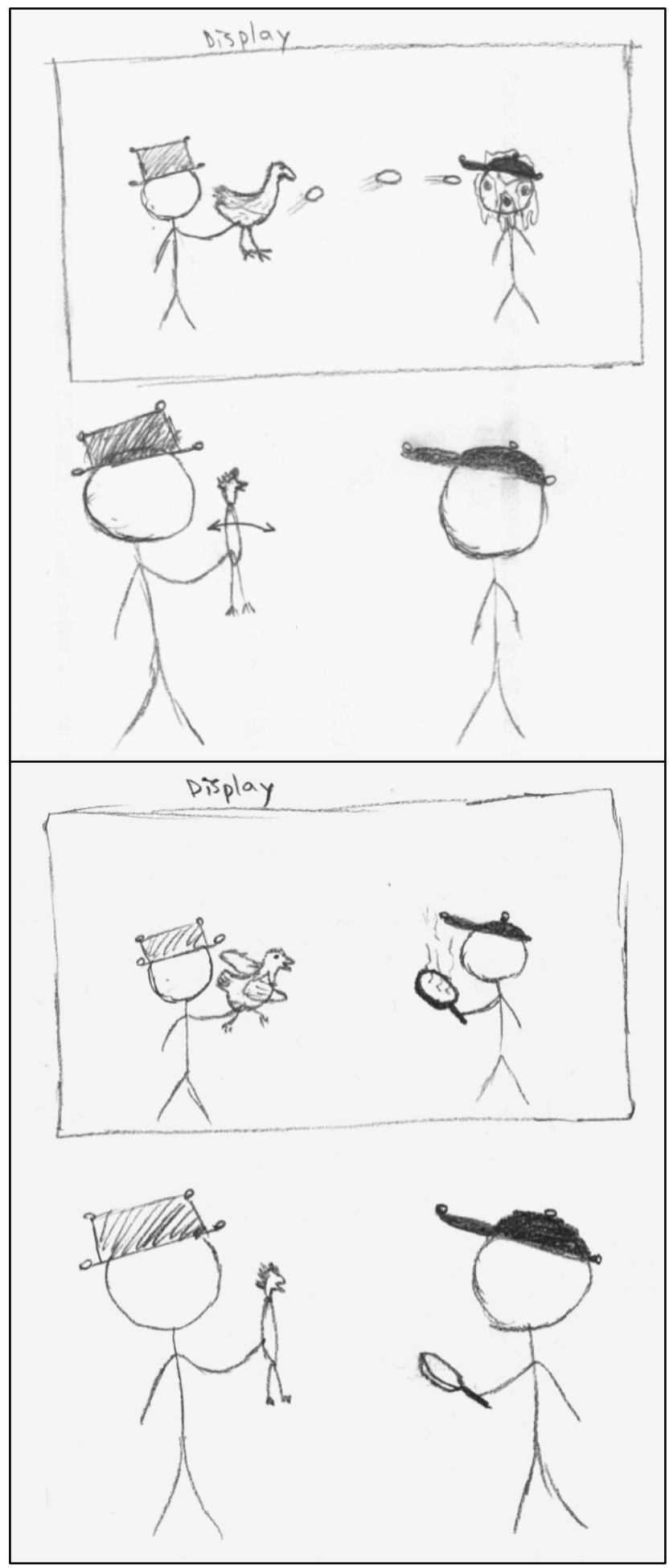

Figure 7: Sketch of an example scenario in JokesOnYou.

heads and hands. Several props will be available to the players, hanging on the sides of the display or inside of boxes on the floor. These props will be tracked by the Vicon system as well.

The game is played by using the props to invoke various augmented reality effects against the opponent. Each prop has a unique virtual effect that can be activated using the prop's perceived physical affordance [7, 10]. The effect will automatically be directed at the other player, who has a chance to block the attack with their own prop. The virtual attacks are purposefully made comical rather than practical; for example, players throw virtual eggs rather than using energy beams against each other.

The player's head and upper torso area is tracked with the marked hat. When a player is attacked with a prop, the attack will occur on their virtual mirror image. For instance, if a virtual egg is thrown at a player, their image on the display will appear to have eggs covering their head. The props are also tracked allowing them to be enhanced with augmented reality, giving the player clues to their attack. For instance, a ball prop will appear as a water balloon on the display.

An example game scenario is described between two players, John and Fred. John picks up a rubber chicken prop that appears as an animated hen on the display. He makes a throwing motion with his arm, sending virtual eggs from the hen to Fred, which hit his head and crack, covering his body with egg drippings. Fred shakes his head back and forth, which appears to throw the bits of virtual egg off his body. While John is still using the chicken to throw eggs, Fred picks up a plastic frying pan prop and uses it to block the virtual eggs, making a virtual omelet. This frightens the hen, which panics and covers John with feathers that stick to him (Figure 7).

JokesOnYou's gameplay will be recorded and can later be played as video clips, posted on video sharing websites or social networking tools.

JokesOnYou is designed to offer players extremely expressive use of physical objects during gameplay and we believe it will it support a very engaging social experience for both players and spectators alike. JokesOnYou's authoring aspect is arguably weak as it is based mostly on unstructured combative gameplay. However, we believe that JokesOnYou's players will pay attention to the theatrical motives of the gameplay. Following, we think that players will engage with the gameplay in a way that will create fun moments for them, as well as for their spectators, practically authoring content.

\section{CONCLUSION AND FUTURE WORK}

In this paper we motivated and argued a new design framework for future games based on the amalgamation of three design themes: content authoring, expressive physical interaction, and social gameplay. To allow us to explore our design framework we presented a unified gaming environment which should support several different game instances. We detailed our efforts of designing three such specific game instances: Social Comics, Film Karaoke and JokesOnYou. For each of the games we described our design, discussed how the gameplay will reflect on our design framework, and presented our current early prototyping efforts.

In the short term we plan to implement fully playable working prototypes of each of the three games we proposed. Once we are satisfied with each prototype, we plan to evaluate it via a thorough user study. This should hopefully allow us to reflect back on our design framework, and to examine whether the design principles presented in this paper are meaningful in practice, and whether they fulfill our design expectations, and support new and unique gameplay experiences. 


\section{REFERENCES}

[1] Bjork, S., Falk, J., Hansson, R., and Ljungstrand, P. 2001. Pirates! Using the physical world as a game board. INTERACT '01 (2001), 423-430

[2] Chaplin, D. J. (1993) Chroma key method and apparatus. U.S. Patent No. 5249039. Washington, DC: U.S. Patent and Trademark Office.

[3] Entertainment Software Association. (2009). 2009 sales, demographic and usage data: Essential facts about the computer and video game industry. \{Online\}. Available at: http://www.theesa.com/facts/pdfs/ESA_EF_2009.pdf, accessed 10 February 2010.

[4] Guo, C., Young, J. E., and Sharlin, E., 2009. Touch and toys: new techniques for interaction with a remote group of robots. In Proceedings of the 27th international conference on Human factors in computing systems. (Boston, MA, USA, 2009), CHI '09. ACM Press, New York, NY, 491500 .

[5] Magerkurth, C., Cheok, D. A., Mandryk, R. L., and Nilsen, T. 2005. Pervasive games: bringing computer entertainment back to the real world. Computers in Entertainment. 3, 3, (Jul. 2005), CIE '05. ACM Press, New York, NY, 4-4.

[6] McCloud, S. Understanding Comics: The Invisible Art. HarperCollins Publishers, Inc. New York, NY, 1994.

[7] Norman, D. A. 1999. Affordance, conventions, and design. In Interactions, 6, 3, (Jun. 1999), ACM Press, New York, NY, 38-43

[8] Reeves, S., Benford, S., O’Malley, C., and Fraser, M. 2005. Designing the spectator experience. In Proceedings of the SIGCHI conference on Human factors in computing systems. (Portland, OR, USA, 2005), CHI '05. ACM Press, New York, NY, 741-750.
[9] Sall, A. and Grinter, R. E. 2007. Let's Get Physical! In, Out and Around the Gaming Circle of Physical Gaming at Home. Computer Supported Cooperative Work (CSCW). 15, 1-2 (Apr. 2007), 199-229.

[10] Sharlin, E., Watson, B., Kitamura, Y., Kishino, F., and Itoh, Y. 2004. On tangible user interfaces, humans and spatiality. Personal and Ubiquitous Computing. 8, 5, (Sept. 2004). 338-346.

[11] U.S. Department of Commerce. (2004). A Nation Online: Entering the Broadband Age. \{Online . Available at: http://www.ntia.doc.gov/reports/anol/NationOnlineBroadba nd04.pdf, accessed 10 February 2010.

[12] Voida, A. and Greenberg, S. 2009. Wii all play: the console game as a computational meeting place. In Proceedings of the 27th international conference on Human factors in computing systems (Boston, MA, USA, 2000). CHI '09. ACM Press, New York, NY, 1559-1568.

[13] Wikipedia. (2010). PlayStation Eye. \{Online $\}$. http://en.wikipedia.org/wiki/PlayStation_Eye, accessed 10 February 2010.

[14] Wikipedia. (2010). PlayStation Motion Controller. \{Online . Available at: http://en.wikipedia.org/wiki/PlayStation_Motion_Controller , accessed 10 February 2010.

[15] Wikipedia. (2010). Project Natal. \{Online\}. Available at: http://en.wikipedia.org/wiki/Project_Natal, accessed 10 February 2010

[16] Wikipedia. (2010). Spore (2008 video game). \{Online\}. http://en.wikipedia.org/wiki/Spore_(2008_video_game), accessed 10 February 2010. 